Cahiers $d u$ MONDE RUSSE

\section{Cahiers du monde russe}

Russie - Empire russe - Union soviétique et États indépendants

$43 / 4 \mid 2002$

Intellectuels et intelligentsia

\title{
Paul Robinson, The White Russian Army in exile
}

\section{Catherine Gousseff}

\section{OpenEdition \\ Journals}

Édition électronique

URL : https://journals.openedition.org/monderusse/4027

DOI : 10.4000/monderusse. 4027

ISSN : $1777-5388$

Éditeur

Éditions de l'EHESS

Édition imprimée

Date de publication : 30 décembre 2002

Pagination : 718-721

ISBN : 2-7132-1796-2

ISSN : $1252-6576$

\section{Référence électronique}

Catherine Gousseff, "Paul Robinson, The White Russian Army in exile », Cahiers du monde russe [En ligne], 43/4 | 2002, mis en ligne le 17 juin 2009, consulté le 03 septembre 2022. URL : http:// journals.openedition.org/monderusse/4027; DOI : https://doi.org/10.4000/monderusse.4027

Ce document a été généré automatiquement le 3 septembre 2022

Tous droits réservés 


\title{
Paul Robinson, The White Russian Army in exile
}

\author{
Catherine Gousseff
}

\section{RÉFÉRENCE}

Paul ROBINSON, The White Russian Army in exile, 1920-1945. Oxford, Oxford University Press, 2002, $257 \mathrm{p}$.

1 Malgré le nombre croissant de travaux consacrés à l'histoire de l'Armée blanche en exil, l'étude de Paul Robinson apporte, à plusieurs titres, un regard nouveau sur le devenir des émigrés militaires depuis leur départ de Russie en 1920 jusqu'à la Seconde Guerre mondiale.

2 Ainsi que son titre le suggère, The White Russian Army in exile est conçu comme une synthèse historique retraçant, tour à tour, les étapes géographiques et les formes d'implantation des groupes de l'Armée blanche à l'étranger, les différents aspects organisationnel, générationnel, socio-culturel, idéologique et politique de l'exil militaire. Deux grandes approches se dégagent de la lecture : l'une relève principalement de l'histoire sociale de cette collectivité dans la diversité des contextes extérieurs rencontrés, l'autre a trait essentiellement à l'histoire politique interne de l'émigration blanche. La première retient plus particulièrement l'attention par les très nombreux éclairages apportés tout au long d'une trame historique bien identifiée : les évacuations collectives au sud de la Russie et l'orientation, par l'armée française, des troupes vers Lemnos, Gallipoli et les camps situés au nord-ouest de Constantinople ; leur redispersion vers la Bulgarie et la Yougoslavie principalement; le nouveau départ, pour certains des réfugiés, vers la Tchécoslovaquie et la France.

La reconstitution de ces parcours concerne donc les membres de l'Armée blanche du Sud évacués vers le Bosphore (et non les troupes ayant quitté le pays via la Pologne ou par l'Extrême-Orient). Parmi les nombreuses spécificités attachées à l'histoire des « évacués » militaires, figure en premier lieu la préoccupation durable de leur dernier 
chef, le général Wrangel, de préserver à tout prix la cohésion de ses troupes en maintenant autant que possible les hommes ensemble, malgré l'adversité d'une situation qui impliquait, compte tenu de la capacité réduite des États d'accueil, la dispersion des réfugiés.

4 À partir de cet objectif, initialement défini à des fins de reconquête, l'auteur analyse comment et dans quelle mesure le haut commandement blanc parvint effectivement à éviter la dislocation de l'armée, quelle fut l'incidence du contrôle qu'il exerça sur les trajectoires des réfugiés militaires et comment cette volonté de contrôle se transforma, assez rapidement, en une vaste action d'assistance sociale et humanitaire. L'historien a retracé, d'une part, les tractations engagées par Wrangel avec les gouvernements des pays d'asile, les résistances rencontrées à la fois sur le plan politique, logistique et matériel et, d'autre part, les parcours des troupes, de plus en plus fragmentées au fur et à mesure de leur redistribution à partir des territoires turcs où elles avaient été déjà réparties entre différents lieux d'accueil. Robinson insiste d'ailleurs sur cette première expérience de l'exil qui, à Gallipoli surtout, constitua l'un des moments forts de la reconstruction des solidarités entre ces hommes après le désarroi qui avait accompagné le départ de Russie. À partir des 100000 hommes évacués en 1920, l'historien a été ainsi amené à suivre les trajectoires des différents groupes, morcelés par la dispersion géographique à travers l'Europe du Sud-Est, mais étroitement apparentés par leur modes de vie, leur insertion souvent précaire dans le monde du travail, leurs modes d'encadrement et d'organisation collective. C'est en effet par le maintien d'une hiérarchie militaire au sein de chacun des groupes, la mise en place de liaisons régulières avec l'État-Major du général Wrangel, la création de différentes formes de solidarité, telles les caisses de secours mutuel, que le sentiment d'appartenance à l'ex-armée a pu être dans une large mesure entretenu, donc préservé. En s'appuyant sur une importante consultation d'archives (en particulier le fonds de l'Union générale militaire russe, ROVS, conservé dans la collection Bahmetev, et le fonds Wrangel conservé à l'institut Hoover), l'auteur reconstitue l'activité déployée par le haut commandement pour placer collectivement les différents contingents dans des grands chantiers, de construction de routes notamment en Yougoslavie, ou des centres industriels comme le bassin minier de Pernik en Bulgarie. Il fait état de quelques initiatives particulièrement réussies comme l'engagement du service topographique de l'armée russe au sein de l'armée yougoslave (une centaine d'officiers), et suit de façon relativement détaillée l'action développée pour la reconversion des hommes à la vie civile, par l'apprentissage professionnel ou, mieux encore, par l'accès à des formations supérieures dans les centres universitaires de Prague, Belgrade ou Bruxelles.

5 L'objectif de non-dislocation de l'armée, partiellement atteint dans la péninsule balkanique, rencontra de nombreux obstacles politiques et logistiques. Robinson rappelle tout d'abord la réaction très hostile du gouvernement français au projet de Wrangel et l'analyse à travers le comportement de l'État-Major français en Turquie. La consultation des archives du Service historique de l'armée de terre lui a permis notamment d'apprécier la diversité avec laquelle les généraux français ont répondu aux injonctions du gouvernement concernant la cessation de la prise en charge des réfugiés militaires et l'encouragement aux rapatriements en Russie. L'historien fait valoir, par exemple, la relative tempérance du général Charpy qu'il oppose au zèle déployé par le général Brousseaud pour encourager le retour des militaires du camp de Lemnos en Russie. Abordant le rôle tenu par les Français dans les vagues de rapatriements qui 
suivirent la fin de la guerre civile, il fournit de nouveaux éléments aux bilans jusque-là effectués.

6 L'État-Major français ne fut pas le seul des acteurs extérieurs à avoir ouvertement prôné le retour des réfugiés militaires en Russie. Dans son chapitre consacré à l'accueil de l'ex-armée en Bulgarie, Robinson analyse toute l'ambivalence de la politique conduite par les autorités de Sofia à son égard dans les années 1921-1923, selon l'évolution des rapports de force sur la scène politique bulgare. Longtemps considérés, dans l'historiographie del'émigration russe, comme des hôtes privilégiés de la Bulgarie, les réfugiés militaires furent en fait l'objet d'instrumentalisations diverses par le gouvernement de Stamboulisky qui chercha rapidement à les disperser en favorisant, notamment, leur rapatriement par le biais du Sovnarod, organisme qu'il soutenait directement. À en juger d'après l'auteur, l'instabilité politique nationale eut, plus que la précarité de la situation économique dans le pays, un effet dissuasif sur les projets d'installation des réfugiés. Néanmoins, les vétérans qui restèrent en Bulgarie furent parmi ceux qui conservèrent le plus durablement les structures et l'état d'esprit qui avaient caractérisé les premières années de l'exil militaire.

7 Orchestré par le haut commandement blanc, le placement des troupes dans l'Europe du Sud-Est fut réorienté à partir de 1923 vers la France, en raison de l'absence de débouchés durables dans les premiers pays d'accueil. Le rôle des généraux blancs dans l'organisation de ces nouvelles migrations, connu jusque-là de façon anecdotique, fait l'objet dans The White Russian Army in exile d'une analyse très rigoureuse et convaincante qui témoigne du degré extrême de contrôle du parcours des vétérans. Observable également en ce qui concerne les civils, celui-ci se manifeste de façon décisive dans les trajectoires des militaires qui, pour la plupart, n'ont pas cessé d'être pris en charge, depuis les rives de la mer Noire jusqu'aux mines de Decazeville ou aux usines d'Argentière.

8 L'encadrement des vétérans n'a pas seulement résidé dans l'organisation des transports et de l'embauche, il a également consisté en une assistance morale, matérielle, culturelle, et Robinson en analyse les diverses articulations par le biais de l'histoire de l'Union générale militaire (ROVS) qu'il reconstitue à travers profils et aspirations de ses dirigeants, mode de fonctionnement et activités, financements, ou encore réseaux et interlocuteurs, qu'ils soient extérieurs ou russes. En abordant ce versant interne de l'histoire de l'émigration militaire, l'historien en vient à étudier la dimension idéologique et les différents aspects de l'engagement politique des Blancs. Il insiste sur la quête difficile d'un compromis entre la posture de "non-prédétermination» (sur la forme à venir du gouvernement de la Russie) prônée par Wrangel, en vertu de laquelle le général proscrivit toute activité politique dans ses rangs, et l'inclination, très largement répandue chez les vétérans, vers le monarchisme. Il passe en revue les différentes tentatives d'infiltration des Blancs en Russie et des Soviétiques dans le ROVS, analyse l'empreinte idéologique du fascisme et du nazisme au sein des réfugiés militaires, retrace l'émergence de nouveaux courants, apportant notamment des éclairages précieux sur les relations entre le ROVS et l'Union nationale de la nouvelle génération (NSNP, futur NTS), en particulier sur la branche terroriste de cette organisation et son entreprise de noyautage du ROVS. L'apport de cette section réside cependant moins dans les faits exposés que dans l'identification de leurs différents acteurs et supporters, selon les générations, les expériences vécues en Russie et en exil - l'historien soulignant, par exemple, des décalages importants dans les mentalités et aspirations entre vétérans russes de la Première Guerre, d'une part (assez fortement représentés dans l'univers associatif de l'émigration), et ceux de la guerre civile, de l'autre. 
9 Cet ouvrage permet d'avoir une vision assez complète, nuancée et distanciée de l'histoire de l'Armée blanche en exil. Il reste pourtant à relever la "part pauvre » de l'étude, qui renvoie au principal dilemme rencontré par les spécialistes de l'émigration russe : elle concerne les origines socio-culturelles de ces milliers de combattants que l'historien, faute de sources, ne peut qu'aborder à gros traits, mais qui décidément manquent à la reconstitution des trajectoires de réfugiés et, plus généralement, à la mise en perspective des identités collectives façonnées par l'exil. 